\title{
BMJ Open Dermoscopy for melanoma detection and triage in primary care: a systematic review
}

\author{
OT Jones, ${ }^{\oplus 1}$ LC Jurascheck, ${ }^{2}$ MA van Melle, ${ }^{1}$ S Hickman, ${ }^{3}$ NP Burrows, ${ }^{4}$ PN Hall, ${ }^{5}$ \\ J Emery, ${ }^{\oplus 1,6}$ FM Walter ${ }^{1,6}$
}

To cite: Jones $0 \mathrm{~T}$, Jurascheck LC, van Melle MA, et al. Dermoscopy for melanoma detection and triage in primary care: a systematic review. BMJ Open 2019;9:e027529. doi:10.1136/ bmjopen-2018-027529

- Prepublication history and additional material for this paper are available online. To view these files, please visit the journal online (http://dx.doi. org/10.1136/bmjopen-2018027529).

Received 26 0ctober 2018 Revised 05 February 2019 Accepted 08 July 2019

Check for updates

(C) Author(s) (or their employer(s)) 2019. Re-use permitted under CC BY. Published by BMJ.

${ }^{1}$ Department of Public Health and Primary Care, University of Cambridge, Cambridge, UK ${ }^{2}$ University of Cambridge School of Clinical Medicine, Cambridge, UK

${ }^{3}$ Norfolk and Norwich University Hospitals NHS Foundation Trust, Norwich, UK

${ }^{4}$ Addenbrooke's Hospital Department of Dermatology, Cambridge University Hospitals NHS Foundation Trust, Cambridge, UK

${ }^{5}$ Addenbrooke's Hospital, Cambridge University Hospitals NHS Foundation Trust,

Cambridge, UK

${ }^{6}$ General Practice and Primary

Care Academic Centre,

University of Melbourne, Carlton, Victoria, Australia

Correspondence to

Dr OT Jones;

otj24@medschl.cam.ac.uk

\section{ABSTRACT}

Objective Most skin lesions first present in primary care, where distinguishing rare melanomas from benign lesions can be challenging. Dermoscopy improves diagnostic accuracy among specialists and is promoted for use by primary care physicians (PCPs). However, when used by untrained clinicians, accuracy may be no better than visual inspection. This study aimed to undertake a systematic review of literature reporting use of dermoscopy to triage suspicious skin lesions in primary care settings, and challenges for implementation.

Design A systematic literature review and narrative synthesis.

Data sources We searched MEDLINE, Cochrane Central, EMBASE, Cumulative Index to Nursing and Allied Health Literature, and SCOPUS bibliographic databases from 1 January 1990 to 31 December 2017, without language restrictions.

Inclusion criteria Studies including assessment of dermoscopy accuracy, acceptability to patients and PCPs, training requirements, and cost-effectiveness of dermoscopy modes in primary care, including trials, diagnostic accuracy and acceptability studies.

Results 23 studies met the review criteria, representing 49769 lesions and 3708 PCPs, all from high-income countries. There was a paucity of studies set truly in primary care and the outcomes measured were diverse. The heterogeneity therefore made meta-analysis unfeasible; the data were synthesised through narrative review. Dermoscopy, with appropriate training, was associated with improved diagnostic accuracy for melanoma and benign lesions, and reduced unnecessary excisions and referrals. Teledermoscopy-based referral systems improved triage accuracy. Only three studies examined cost-effectiveness; hence, there was insufficient evidence to draw conclusions. Costs, training and time requirements were considered important implementation barriers. Patient satisfaction was seldom assessed. Computer-aided dermoscopy and other technological advances have not yet been tested in primary care. Conclusions Dermoscopy could help PCPs triage suspicious lesions for biopsy, urgent referral or reassurance. However, it will be important to establish further evidence on minimum training requirements to reach competence, as well as the cost-effectiveness and patient acceptability of implementing dermoscopy in primary care.

Trial registration number CRD42018091395.

\section{Strengths and limitations of this study}

- This study systematically reviews the published evidence for dermoscopy use by primary care physicians in primary care settings, including studies of acceptability and cost-effectiveness, as well as diagnostic accuracy studies.

- The use of a broad search strategy across multiple databases enabled us to identify 23 studies whose findings examine dermoscopy use in primary care clinical practice.

- The included studies were of varying quality.

- Due to the heterogeneity of the included papers, we were not able to undertake any meta-analysis; instead, we performed a narrative synthesis.

\section{INTRODUCTION}

Worldwide malignant melanoma is the 15 th most common cancer. ${ }^{1}$ Melanoma has one of the fastest rising incidence rates of any cancer, and among white populations incidence has quadrupled over the last 30 years. In the UK this is projected to rise by a further $7 \%$ between 2014 and 2035, reflecting increasing exposure to the main risk factor, ultraviolet radiation. ${ }^{2}$ There were nearly 300000 new cases of melanoma worldwide in $2018 .{ }^{1}$

Primary care (the first point of contact for patients in the healthcare system, usually community-based) can play an important role in improving outcomes for patients with melanoma. More accurate triage of suspicious pigmented skin lesions could lead to more prompt diagnosis of melanoma at earlier stages and improved outcomes, and reduce unnecessary biopsies or referrals. Most people diagnosed with cancer first present in primary care, ${ }^{3}$ where primary care physicians (PCPs) need to distinguish rare melanomas from common benign lesions using clinical history taking and visual inspection, aided by checklists such as the 7-point checklist as recommended in the 2015 National Institute for Health and Care Excellence guidelines 
for suspected cancer. ${ }^{4}$ Various technologies may also have a role in assisting triage of suspicious skin lesions, including mobile phone applications, ${ }^{5}$ reflectance confocal microscopy, ${ }^{6}$ optical coherence tomography, ${ }^{7}$ computer-aided diagnosis, ${ }^{8}$ high-frequency ultrasound ${ }^{9}$ and dermoscopy. ${ }^{10}$

Dermoscopy (also referred to as dermatoscopy or epiluminescence microscopy) is a non-invasive technique using a hand-held magnifier and incident light, which may be polarised to reduce reflection, to reveal subsurface structures. Dermoscopy performed by trained specialists is more sensitive and specific in classifying skin lesions than clinical examination with the naked eye alone. ${ }^{411}$ Dermatologists and some international guidelines recommend PCPs use dermoscopy ${ }^{12}$; however, when used by untrained or less experienced clinicians, accuracy can be no better than inspection alone, ${ }^{13}$ and there is a danger of increased excisions, over-referral or false reassurance. It takes time to train clinicians to use dermoscopy, and PCP training dropout rates have been shown to be high. ${ }^{14}{ }^{15}$ For these reasons dermoscopy is not currently recommended for use by PCPs in the UK, ${ }^{4}$ although it is used routinely by PCPs in Australia, ${ }^{16}$ which has the highest incidence of melanoma worldwide. Some digital dermoscopy devices exist, a few of which incorporate computer-aided diagnosis, but they are expensive, and while showing better sensitivity even in expert hands many have lower specificity than clinicians alone. ${ }^{17}$ However, recent research suggests computer-aided diagnostic tools have the potential to exceed the diagnostic performance of dermatologists. ${ }^{18}$

A Cochrane review of dermoscopy has recently been published and examines the diagnostic accuracy of dermoscopy, with and without visual inspection, for the detection of cutaneous invasive melanoma and intraepidermal melanocytic variants in adults. ${ }^{19}$ Our systematic review has a broader aim, focusing on the first presentation of suspicious skin lesions in primary care and whether dermoscopy and dermoscopy-related technologies, with suitable training, can be used accurately and effectively to triage suspicious skin lesions at this point in the healthcare pathway. We considered various types of dermoscopy technologies, including hand-held dermoscopy, computer-aided/digital dermoscopy devices and novel teledermoscopy approaches (ie, referral using electronic dermoscopy images or video). In addition to data on the diagnostic accuracy of dermoscopy, we looked for data on the practical challenges to implementing dermoscopy in primary care, including utility, acceptability to patients and PCPs, training requirements, and cost-effectiveness.

\section{METHODS}

This systematic review was conducted in accordance with the Preferred Reporting Items for Systematic Reviews and Meta-Analyses (PRISMA) guidelines,${ }^{20}$ and the protocol was registered with PROSPERO prior to conducting the review. ${ }^{21}$ All aspects of the protocol were reviewed by senior faculty from the CanTest Collaborative (www. cantest.org).

We searched the MEDLINE, EMBASE, Cochrane Central, Cumulative Index to Nursing and Allied Health Literature, and SCOPUS databases using keywords related to dermoscopy, melanoma and primary care, without language restrictions, from 1 January 1990 to 31 December 2017. We also manually searched the reference lists of included studies. We included all types of study design as we anticipated that there would be few relevant randomised controlled trials (RCTs) or diagnostic accuracy studies performed in primary care, and we aimed to find additional qualitative evidence on barriers to the use of dermoscopy which may be found in non-RCT study designs. We chose to start the search from 1990 as this was when the earliest dermoscopy-related research emerged. We considered published evidence from any international healthcare system and whether it could be interpreted and applied to primary care settings, including the extent to which data collected from specialist clinic settings could be applied to the lower-prevalence primary care population.

We included all studies which provide evidence around test accuracy, utility, acceptability to patients and PCPs, training requirements, and cost-effectiveness of dermoscopy modes in primary care, including trials, diagnostic accuracy and acceptability studies. As our interest was in the use of dermoscopy by generalist clinicians, we included all studies reporting PCP use of dermoscopy; studies of secondary care physicians who were not trained in dermoscopy were assessed for the applicability of their study to answer the research question. We excluded studies that were based in any clinical setting other than at the first assessment of suspicious skin lesions, and any studies that were not considered primary studies.

Following duplicate removal, one author (OJ) screened titles and abstracts to identify studies which fitted the inclusion criteria. Of the titles and abstracts $10 \%$ were checked by two other authors ( $\mathrm{LJ}$ and $\mathrm{SH}$ ), and interassessor reliability was excellent, with disagreement for only 1 out of the 100 papers checked. Any disagreements were discussed by the core research team (OJ, LJ, SH, FMW) and a consensus reached. At least two reviewers (OJ, LJ, SH, MvM, FMW) independently assessed each fulltext article for the possibility of inclusion in the review. Any disagreements were resolved by consensus-based discussion.

Data extraction was undertaken by two reviewers independently (OJ, LJ, FMW) and summarised using descriptive tables, discussion and consensus. We chose to extract only reported outcomes from the included papers, without calculating further quantitative measures of diagnostic accuracy from their data, unless already reported. Due to the heterogeneity of the included papers, we were not able to undertake any meta-analysis; instead, we chose to perform a narrative synthesis.

Risk-of-bias assessment was undertaken for each fulltext paper by two independent researchers (OJ, LJ) using 


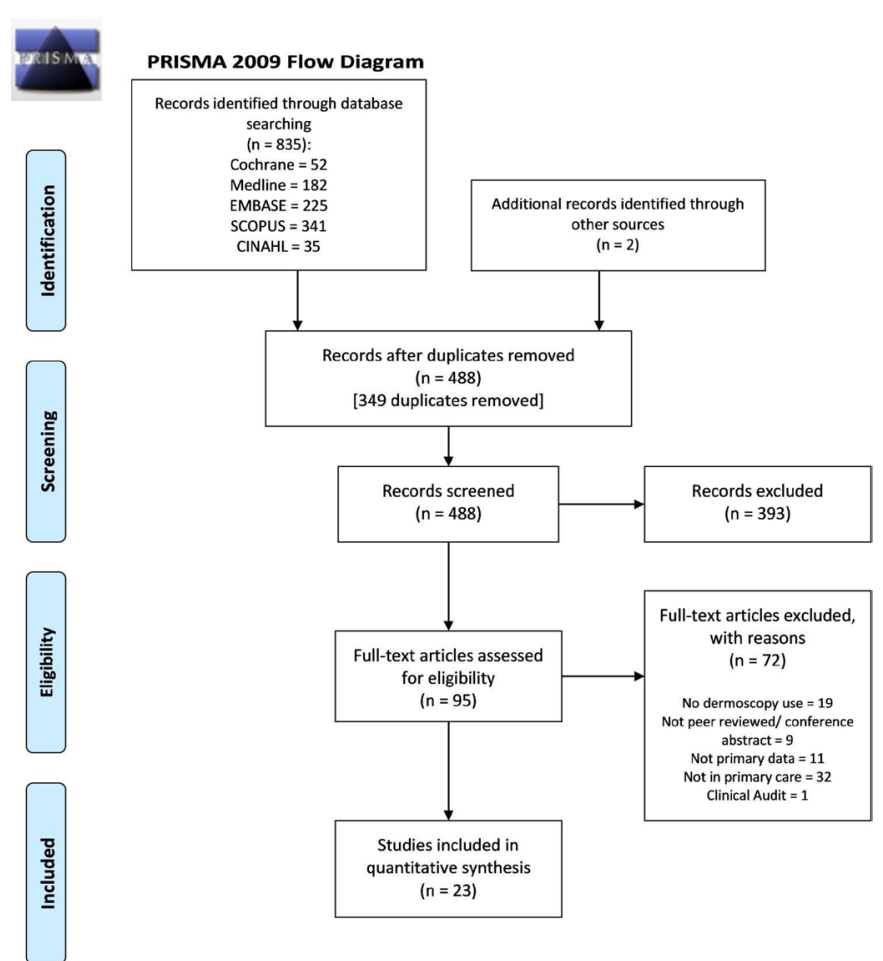

Figure 1 PRISMA flow diagram for the studies included in the review. CINAHL, Cumulative Index to Nursing and Allied Health Literature; PRISMA, Preferred Reporting Items for Systematic Reviews and Meta-Analyses.

the Joanna Briggs Institute (JBI) critical appraisal tools. ${ }^{22}$ These tools incorporate various critical assessments for different study designs, including patient selection, randomisation, data collection and analysis. As assessments for different study designs had varying denominators, the score was converted to a percentage and classified as high, medium and low risk to aid clarity of presentation and interpretation. Although the studies demonstrated a wide range in quality, no studies were excluded based on their risk-of-bias assessment. Full details of our review question, search strategy, inclusion/exclusion criteria, methodology for data extraction, risk-of-bias assessment and outcomes extraction are described in online supplementary appendices 1 and 2, as well as a full list of excluded studies (online supplementary appendix 3).

\section{Patient and public involvement}

Our long-standing collaborator, Mrs Margaret Johnson, is a patient advocate. She commented regularly on the study from its conception, including aspects of the research question, outcome measures and study design. There was no patient recruitment required for this study. The results will be disseminated to patient advocates, groups and relevant charities.

\section{RESULTS}

Figure 1 shows the study PRISMA diagram. There were 837 studies identified, of which 349 were duplicates. Ninety-five articles underwent full-text review and 23 met the inclusion criteria. ${ }^{1423-44}$ These 23 articles reported data relating to 49769 lesions and 3708 PCPs.

Table 1 provides a summary of the study characteristics for included studies. We included three RCTs, two sequential intervention trials (SIT), nine diagnostic accuracy studies, two cohort studies, two case series, one case-control study and four PCP surveys. Table 1 also visually summarises the practitioner and patient populations reported in the studies and highlights the paucity of studies reporting PCPs using dermoscopy with primary care patients ( 5 out of 16 ). Studies of teledermoscopy-based referral systems were more frequently set in primary care, with six out of seven studies involving primary care clinicians and primary care patients. Overall, 16 of the 23 papers reported studies of PCPs, but only 11 papers reported studies involving primary care patients.

Table 2 summarises the outcome measures of each included study, grouped into accuracy and reliability outcomes and implementation outcomes, and shows the heterogeneous nature of the reported outcomes. The accuracy and reliability outcomes were diverse; 12 papers reported sensitivity and specificity, 8 reported diagnostic accuracy or area under the curve, 5 reported positive and negative predictive values, 14 reported the proportion of correct decisions, 4 reported the number needed to excise, and 5 reported the biopsy rate. The implementation outcomes were less numerous but also quite diverse: 4 papers reported on PCP opinions, 3 performed cost-effective analyses, 2 looked at response times for teledermoscopy services, 2 looked at image quality for teledermoscopy, and 1 assessed patient satisfaction.

Risk-of-bias outcomes from the JBI critical appraisal tools are included in table 2 , demonstrating a wide range in quality across the studies. No studies were excluded based on the risk-of-bias assessment.

Tables 3 summarises the diagnostic accuracy results, with the studies grouped into RCTs and SITs, non-RCT diagnostic accuracy studies, and survey studies. Among the RCTs and SITs, Argenziano et $a l^{23}$ Koelink et al, ${ }^{24}$ Rosendahl et $a t^{43}$ and Menzies $e t a l^{14}$ found that dermoscopy reduced the number needed to excise to diagnose a melanoma. Ferrándiz $e t a l^{26}$ evaluated the impact of adding dermoscopic images to the standard teledermatology referral system and found that it improved accuracy and confidence in diagnosing skin lesions.

Most of the studies were non-RCT diagnostic accuracy studies. These showed increased diagnostic accuracy with the use of dermoscopy in primary care 283335434 or in teledermoscopy-based referral systems. ${ }^{34-37}$ Some studies suggested this was due to improved ability to identify benign lesions when using a dermatoscope. ${ }^{27} 313244$ All studies that assessed the effect of training found that it improved diagnostic accuracy compared with minimal or no training. ${ }^{25-33} 44$ There was evidence that use of dermoscopy without training displayed similar diagnostic accuracy to naked-eye examination. ${ }^{33}$ Menzies et $a l^{29}$ showed that a dermoscopy-related technology, SolarScan, 


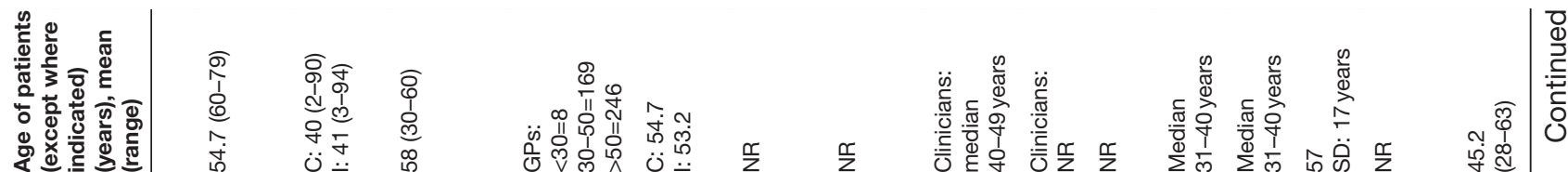

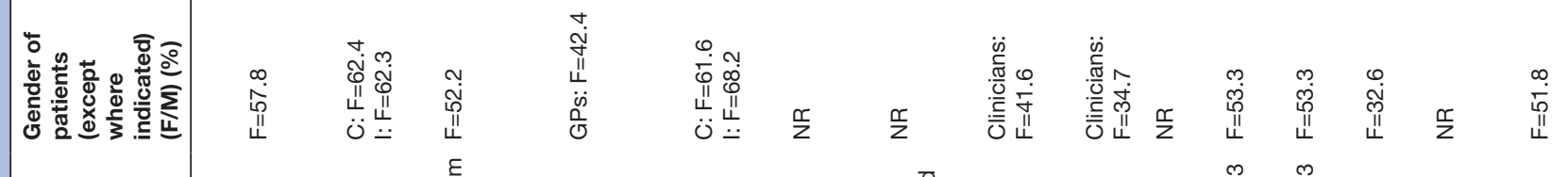

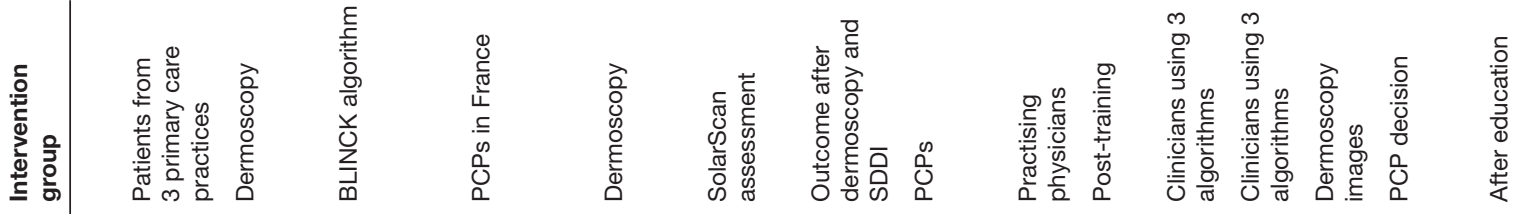

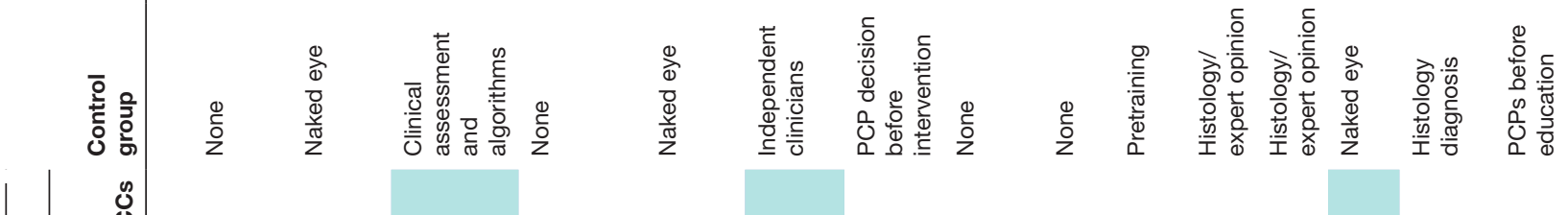
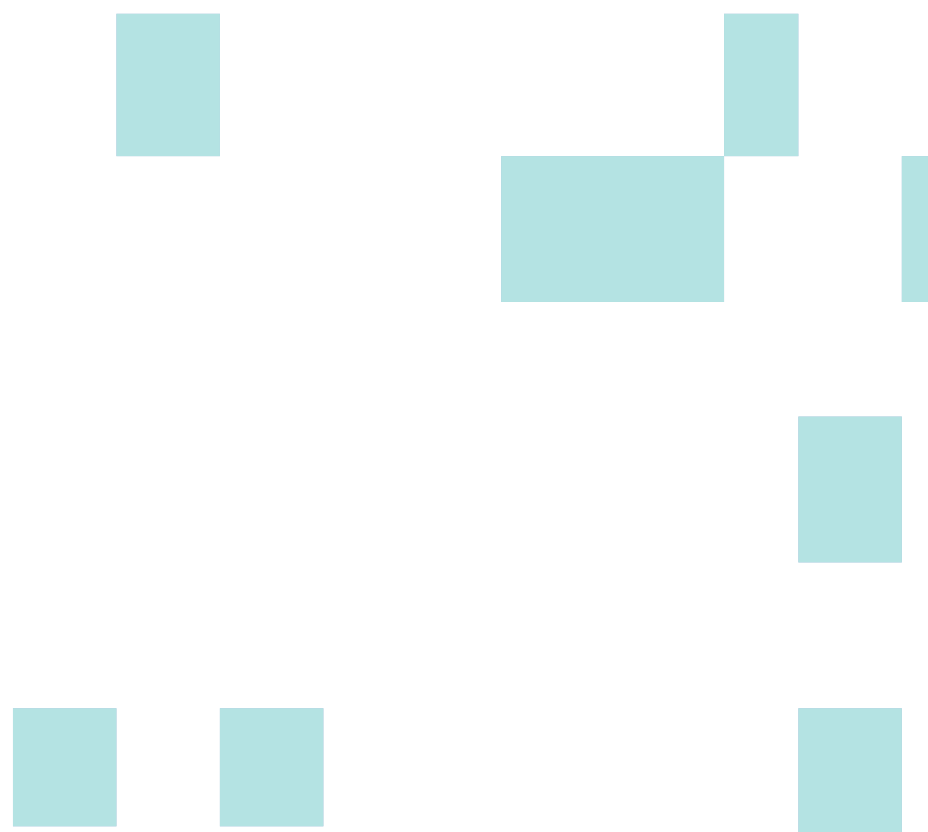
흔
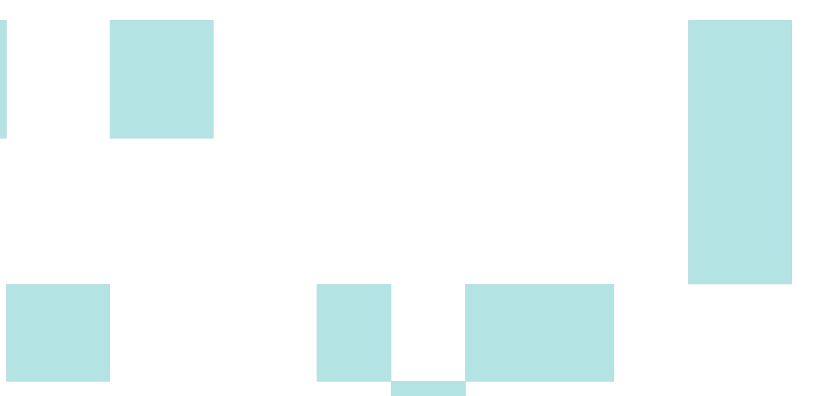
品 -

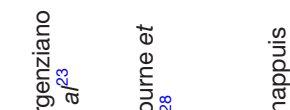
等 


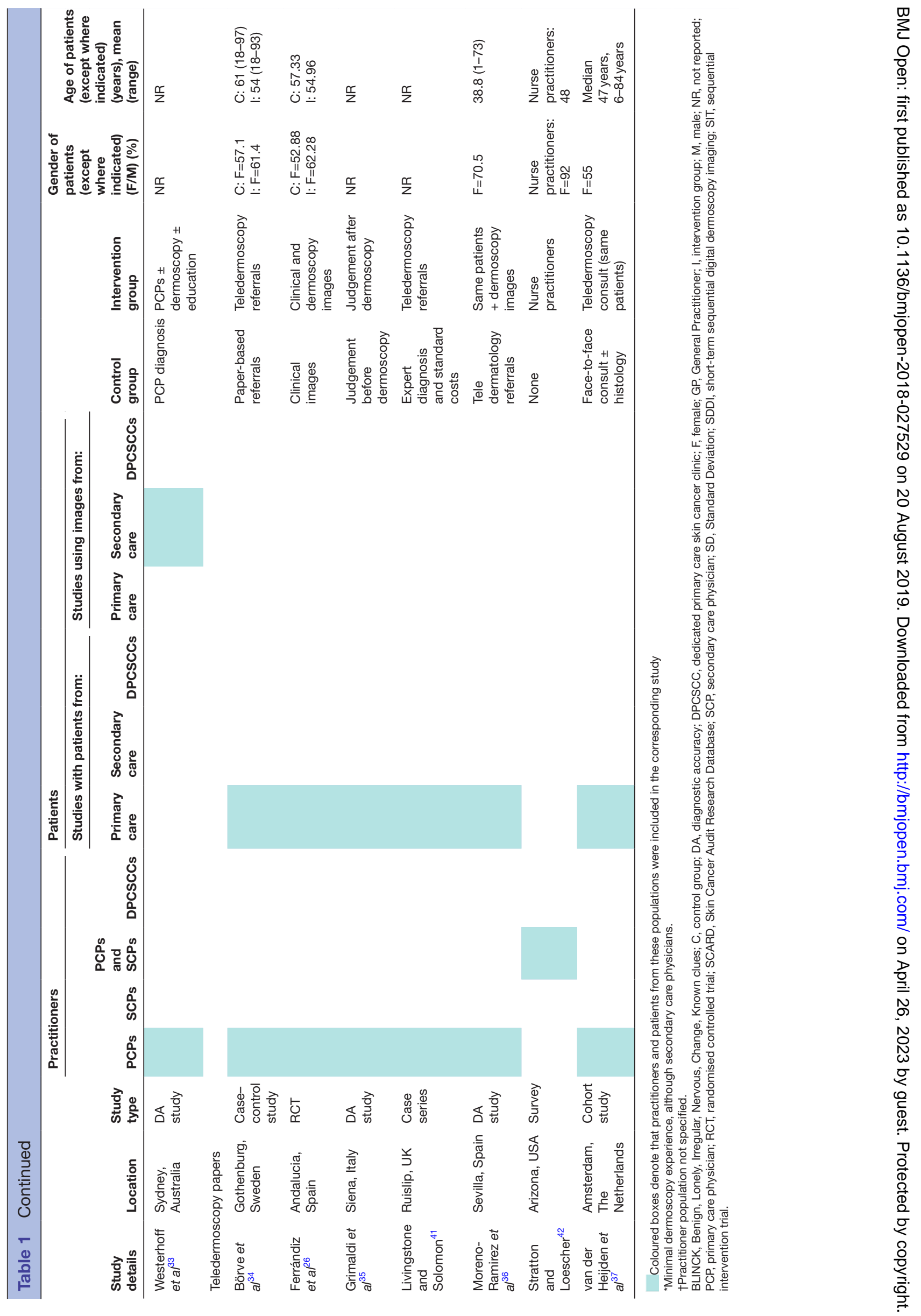




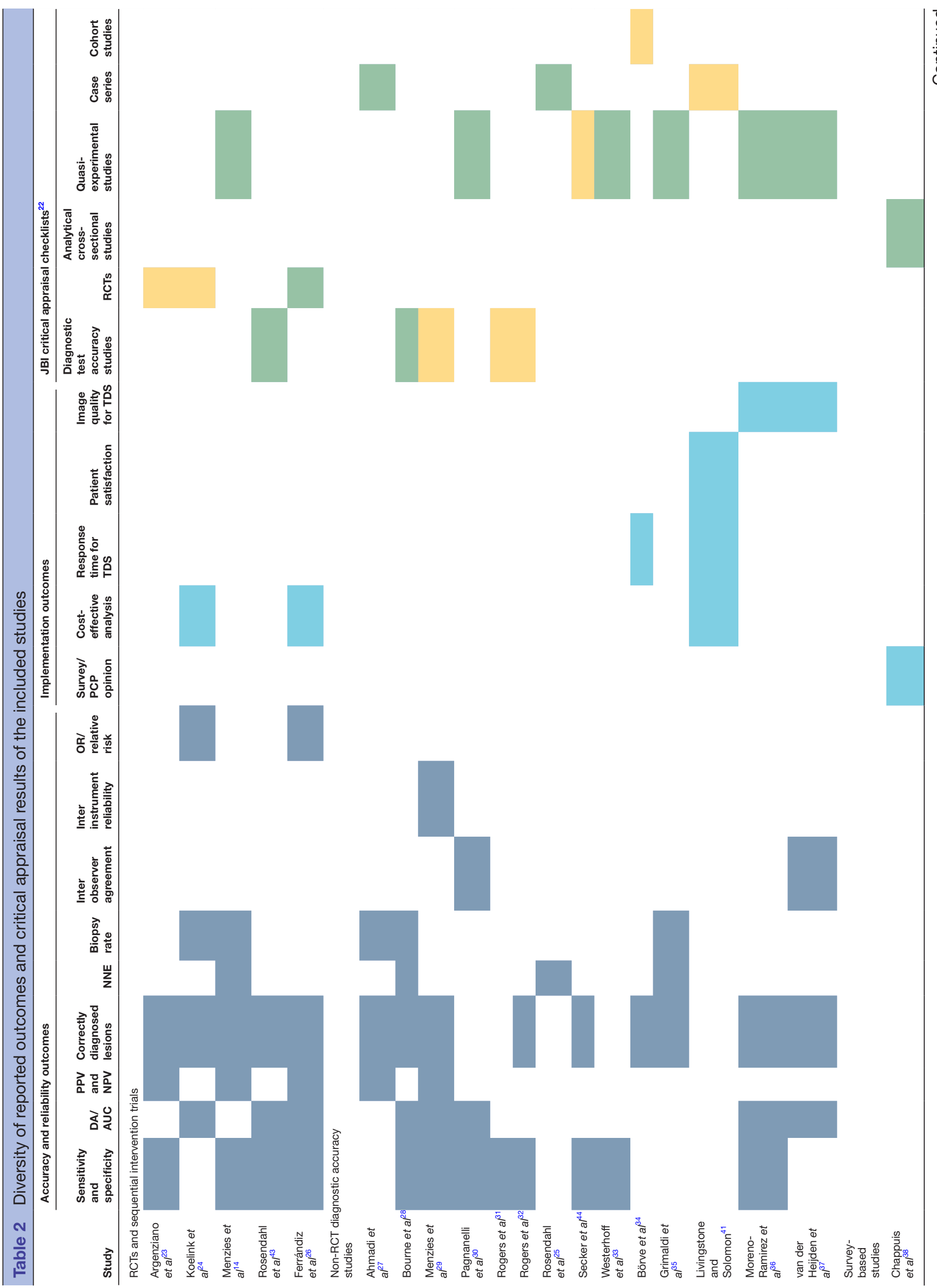

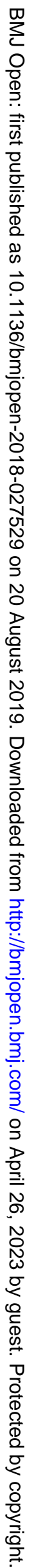




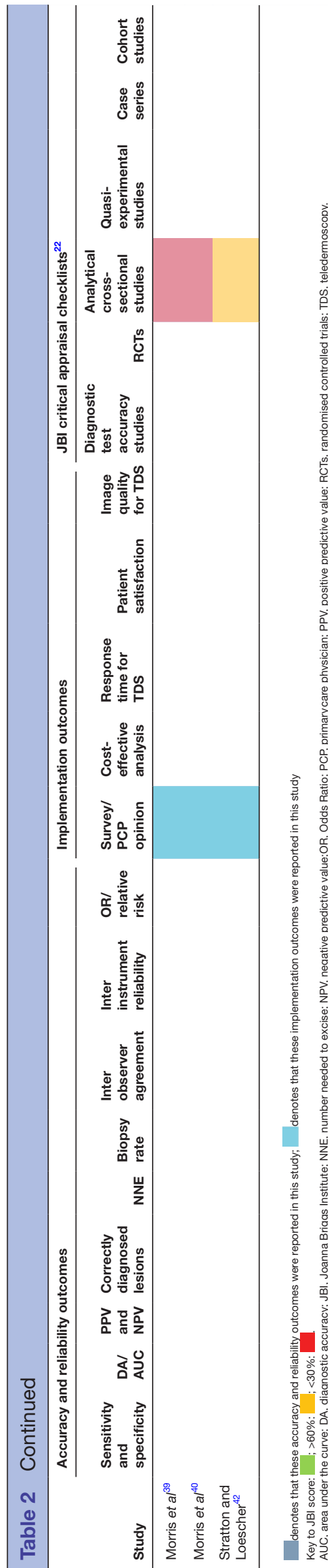

had higher sensitivity than PCPs, although this was a non-significant finding.

Table 4 summarises findings from the studies which investigated barriers and facilitators to implementing dermoscopy in primary care. Training requirements, cost of equipment and the time taken to perform dermoscopy were the most important barriers identified from the studies. However, for each barrier there were some papers that described it as a facilitator instead. Three papers performed cost-effective analyses of dermoscopy ${ }^{24}$ and teledermoscopy, ${ }^{26}{ }^{41}$ and none found a significant cost-effective advantage. The main facilitators identified to the use of dermoscopy in primary care were reduced referrals, early detection of melanoma, and reduced patient and physician anxiety.

\section{Patient and PCP attitudes and acceptance of dermoscopy}

Several papers assessed PCP attitudes to dermoscopy through questionnaires. Stratton and Loescher ${ }^{42}$ found that nurse practitioners in the USA did not widely use dermatoscopes; however, they thought that dermoscopy would have a positive impact and would be willing to use mobile teledermoscopy if they received training. Morris $e t a l^{99}$ found that dermoscopy use among US physicians and doctors of osteopathic medicine was associated with seeing higher numbers of patients and with higher confidence in diagnosing skin lesions. Chappuis $e t a l^{88}$ found that dermoscopy use among French general practitioners (GPs) was associated with being older and male, and that only $8 \%$ of respondents had access to a dermatoscope. Livingstone and Solomon's ${ }^{41}$ survey was the only one that assessed patient acceptability; they reported that $97 \%$ of patients from one general practice in Greater London were satisfied with the teledermoscopy service and $100 \%$ would recommend it.

\section{DISCUSSION}

\section{Principal findings}

Only a small number of studies have examined the use of dermoscopy or dermoscopy-related technologies in the primary care setting. These studies were all set in Europe, the USA and Australia, and due to their heterogeneous nature we were not able to synthesise the findings. Nevertheless, our review found that, with appropriate training, dermoscopy in primary care is more accurate than naked-eye examination, with improvements in sensitivity and specificity and number needed to excise. Furthermore, there was some evidence that teledermoscopy-based referral systems improve triage accuracy compared with paper-based or macroscopic image-based referral systems. The limited evidence did not show a significant cost-effectiveness benefit for either dermoscopy or teledermoscopy, although dermoscopy appears to lead to a reduction in unnecessary referrals and excisions. Importantly, the review also suggests that PCPs are receptive to incorporating dermoscopy into their routine practice, although they recognised ongoing implementation 


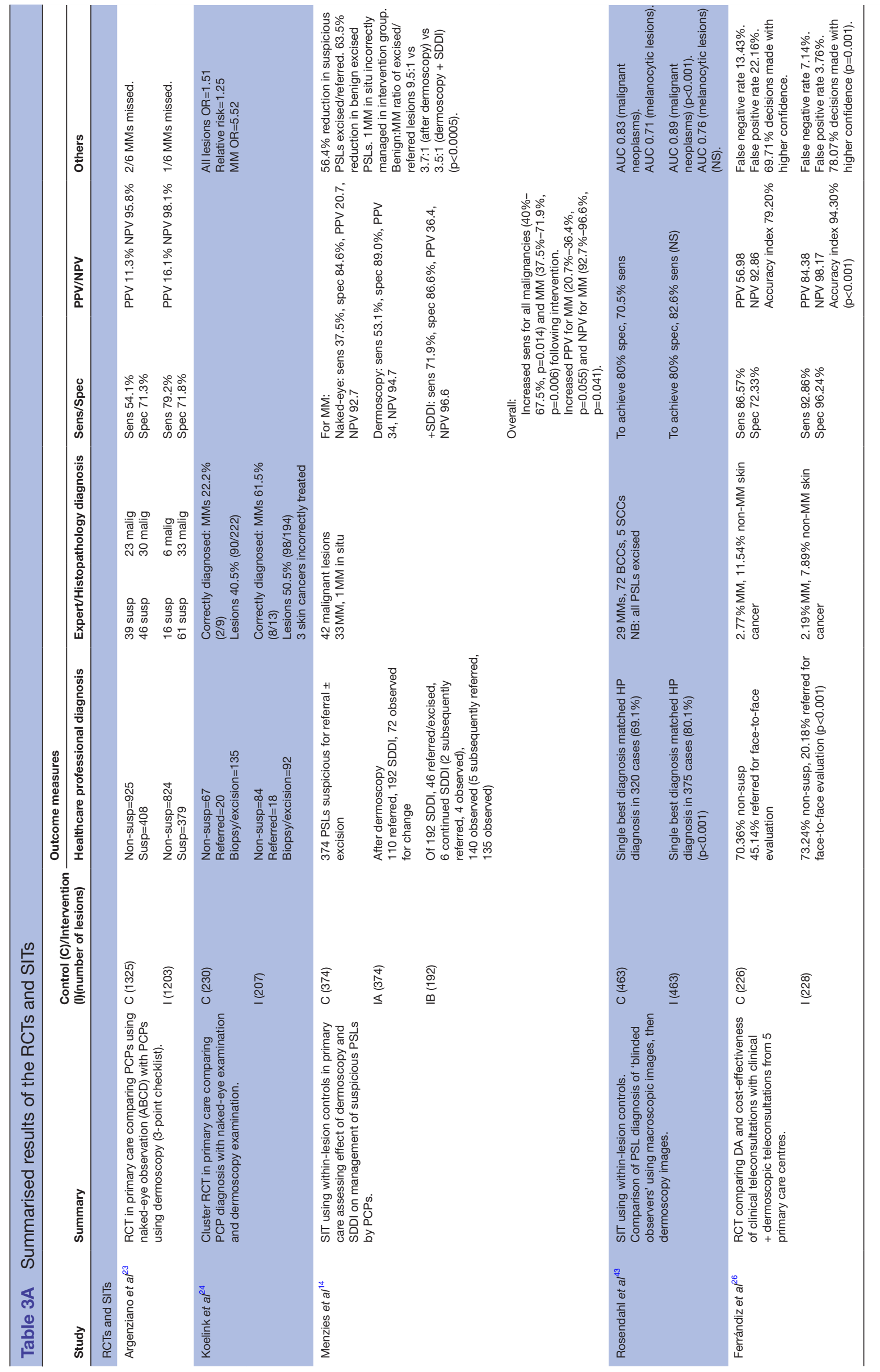




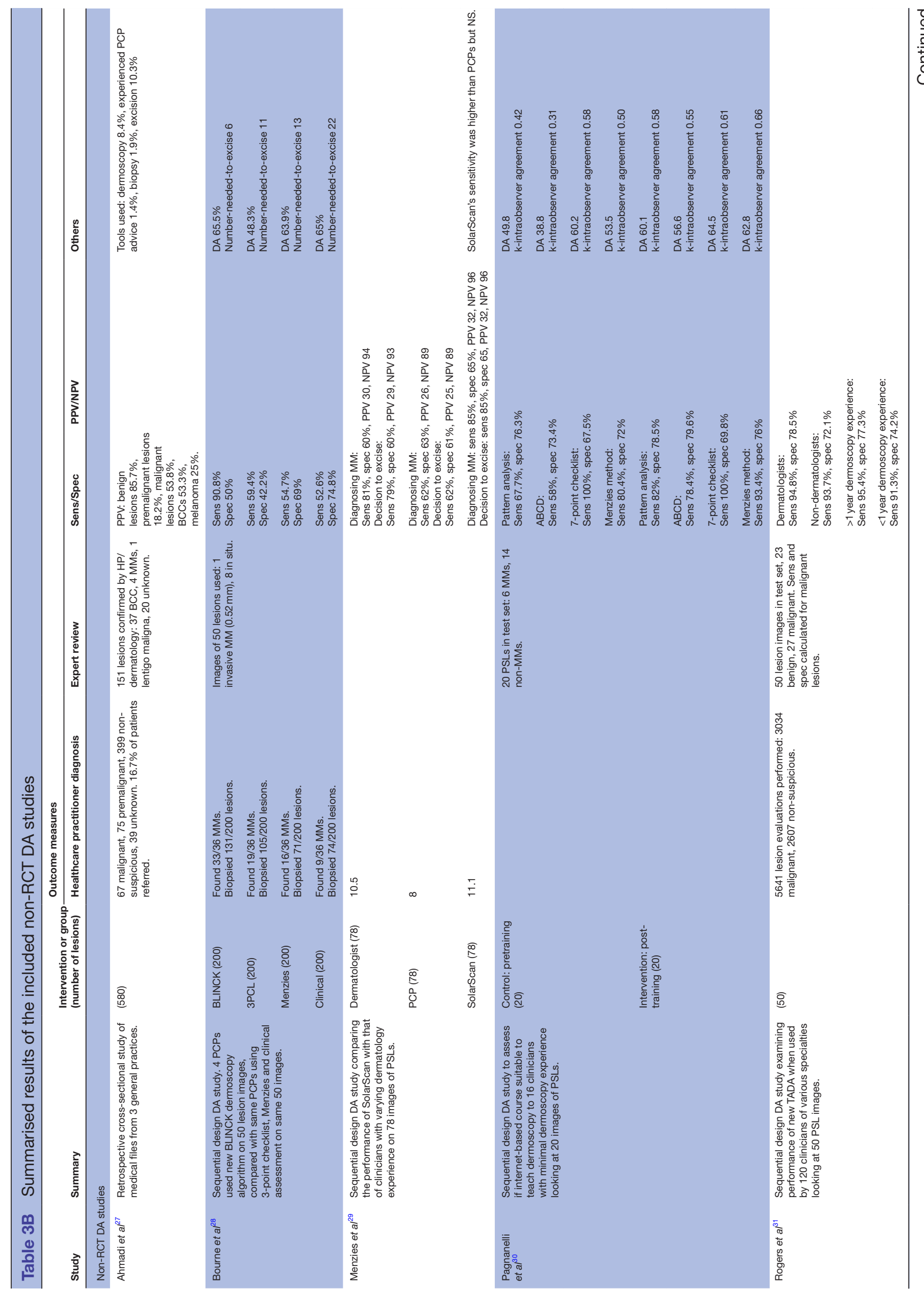




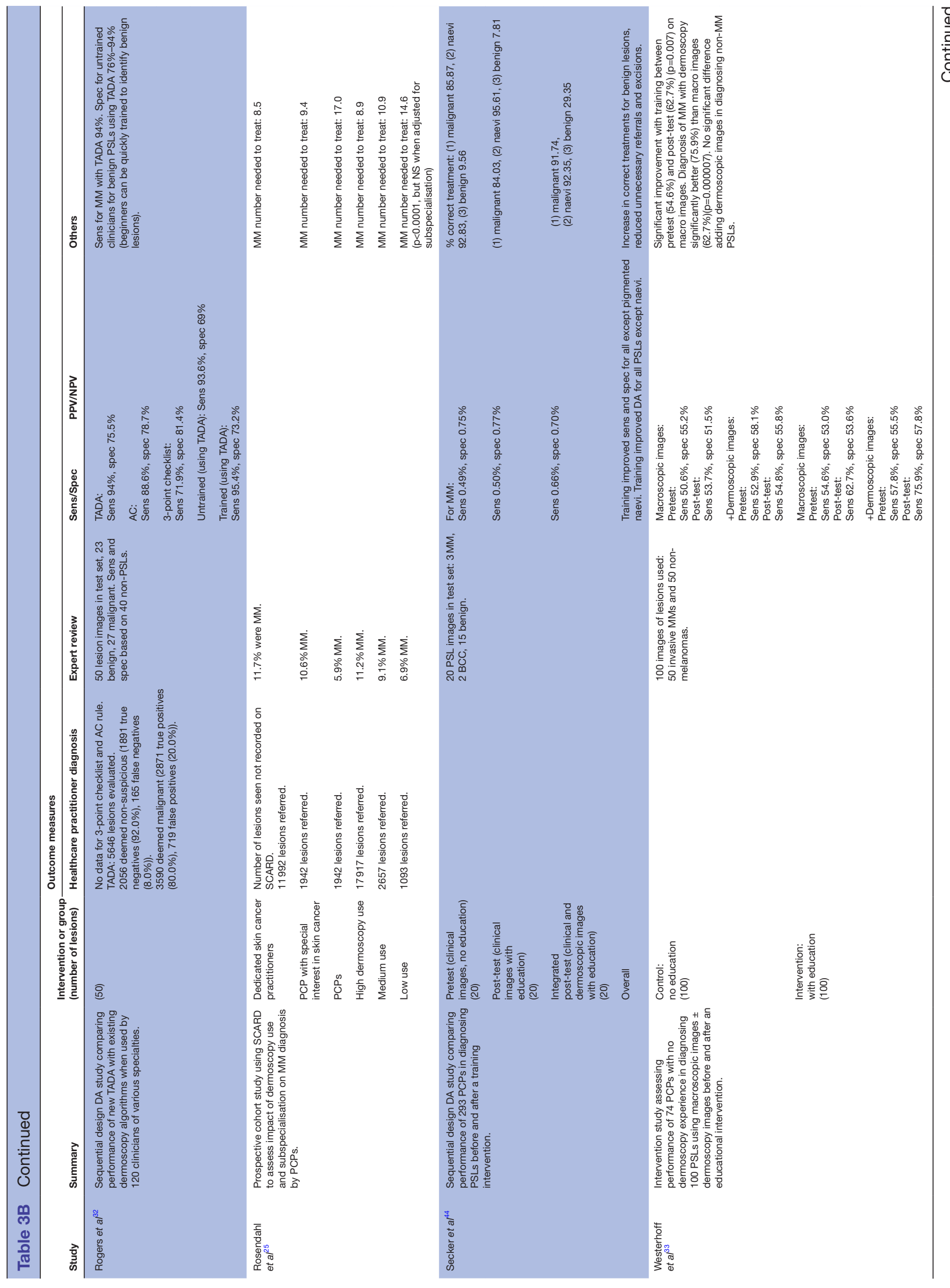




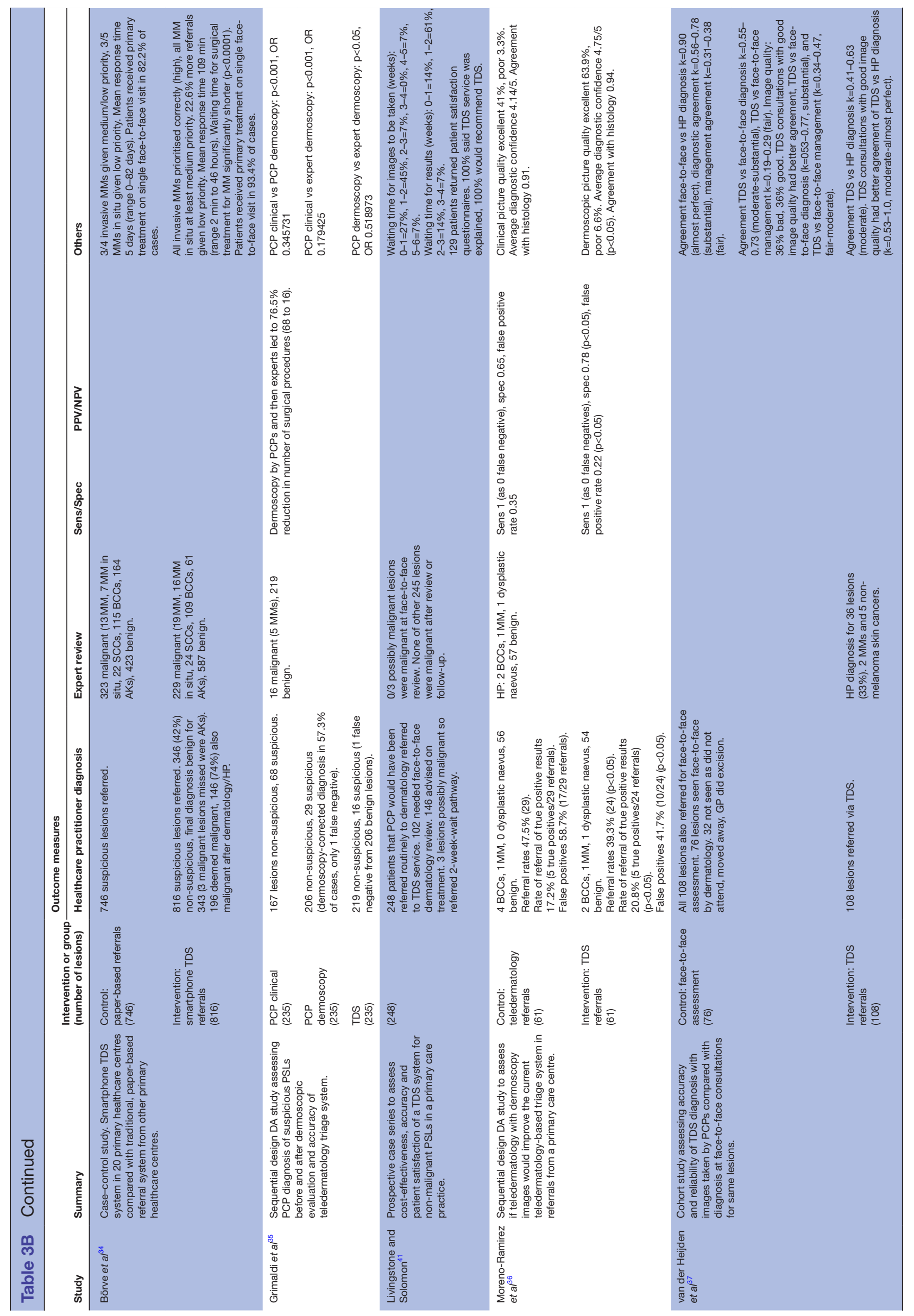

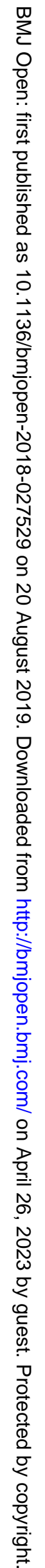


barriers, particularly around training, time requirements and technology costs.

\section{Comparison with other studies}

Our review suggests that dermoscopy in primary care is more accurate than naked-eye examination, supporting the findings from a previous review of dermoscopy for melanoma detection specifically in primary care published in 2012. ${ }^{45}$ A recently published Cochrane review of dermoscopy for the diagnosis of melanoma has also concluded that, although data to support dermoscopy use in primary care are limited, 'it may assist in triaging suspicious lesions for urgent referral when employed by suitably trained clinicians' ${ }^{19}$ Our review also suggests that training PCPs in dermoscopy improves diagnostic accuracy. Again, this finding is supported by the recent Cochrane review which also suggests that 'formal algorithms may be of most use for dermoscopy training purposes and for less expert observers, however reliable data comparing approaches using dermoscopy in-person are lacking'. ${ }^{19}$ Previous reviews have shown that using dermoscopy without training was no more accurate than naked-eye examination alone. ${ }^{13} 19$ However, we were not able to identify the optimal length of training needed to train PCPs to use dermoscopy accurately, although studies of the effect of training on dermatologist diagnostic performance have shown improvement after between 2 days ( 6 hours of training per day) ${ }^{46}$ and 10 weeks (comprising 6 workshops of $4-6$ hours). ${ }^{47}$ PCPs are likely to need short training courses, preferably with regular updates, as one of the few RCTs examining the impact of dermoscopy on the management of pigmented lesions in primary care reported a high dropout rate of GPs from the 20 hours of online trainingrequired for that study. ${ }^{14}$

It is important to note that the performance of dermoscopy in specialist clinics is not directly translatable as evidence for the performance of dermoscopy in primary care settings. A spectrum effect or spectrum bias is often observed when tests developed in one population are then used on another population. For example, the secondary care population is a referred population and has a higher prevalence of the condition being tested than primary care populations. This means that a diagnostic test, such as dermoscopy, will perform differently in the primary care population with the lower prevalence of the condition, compared with the secondary care population. ${ }^{48}$ The direction of effect is not consistent across tests and conditions; hence, to establish the performance of tests among the non-referred population in primary care, they need to be evaluated in a primary care population. This review has therefore aimed to examine existing evidence for dermoscopy use in primary care settings.

Our review suggests that a range of PCPs, including nurse practitioners in the USA, and PCPs in the USA and France, hold positive views about incorporating dermoscopy into their routine practice. Evidence from Australia supports these views and demonstrates that 
Table 4 Barriers and facilitators to implementation of dermoscopy and teledermoscopy

\begin{tabular}{|c|c|c|c|c|}
\hline Aspect & Quoted as barrier in: & Type of study & Quoted as facilitator in: & Type of study \\
\hline \multirow[t]{2}{*}{ Training requirements } & Chappuis et $a l^{38}$ & Survey & Pagnanelli et $\left.a\right|^{30}$ & DA study \\
\hline & Morris et $a l^{40}$ & Survey & & \\
\hline \multirow[t]{3}{*}{ Cost $^{*}$} & Chappuis et al ${ }^{38}$ & Survey & Koelink et $\left.a\right|^{24 \star}$ & $\mathrm{RCT}$ \\
\hline & Morris et $a /^{39}$ & Survey & Rosendahl et $a^{25}$ & Cohort study \\
\hline & Moreno-Ramirez et $a^{36}$ & DA study & Ferrándiz et $a^{26 *}$ & $\mathrm{RCT}$ \\
\hline \multirow[t]{3}{*}{ Time consumption } & Chappuis et $a l^{38}$ & Survey & Börve et $a l^{34}$ & Case-control \\
\hline & Moreno-Ramirez et a/ ${ }^{36}$ & DA study & & \\
\hline & van der Heijden et $\left.a\right|^{37}$ & Cohort study & & \\
\hline $\begin{array}{l}\text { Reimbursement for offering } \\
\text { dermoscopy services (in USA) }\end{array}$ & Morris et $a^{39}$ & Survey & & \\
\hline \multirow{3}{*}{ Reduced referrals } & & & Koelink et $\left.a\right|^{24}$ & $\mathrm{RCT}$ \\
\hline & & & Börve et $a l^{34}$ & DA study \\
\hline & & & Moreno-Ramirez et $\left.a\right|^{36}$ & DA study \\
\hline Early detection of melanoma & & & Chappuis et $a l^{38}$ & Survey \\
\hline Reduced patient anxiety & & & Chappuis et $a^{\beta 8}$ & Survey \\
\hline \multirow[t]{3}{*}{ Reduced physician anxiety } & & & Chappuis et $a /^{\beta 8}$ & Survey \\
\hline & & & Moreno-Ramirez et a $a^{36}$ & DA study \\
\hline & & & Menzies et $a l^{14}$ & DA study \\
\hline
\end{tabular}

*Based on studies where a cost-effective analysis was undertaken. $\mathrm{DA}$, diagnostic accuracy; RCT, randomised controlled trial.

a wide range of PCPs are able to incorporate dermoscopy into their routine clinical practice. ${ }^{16}$ Only a small number of cost-effectiveness studies met our review criteria. They all assessed dermoscopy and teledermoscopy from a healthcare perspective, and only reported on short-term costs resulting from dermoscopy or non-dermoscopy approaches. None reported a significant cost-effectiveness benefit for dermoscopy ${ }^{24}$ or teledermoscopy,${ }^{26}$ in the primary care setting, although they recommended the technologies as potentially useful tools. An English RCT of another diagnostic aid (MoleMate, incorporating SIAscopy) in primary care ${ }^{49}$ also reported equivocal findings on cost-effectiveness, as the device, similar in accuracy to systematic application of the 7-point checklist, resulted in increased referrals from primary care. ${ }^{49} 50$

Interestingly, no papers reporting the use of dermoscopy smartphone applications ('apps') for automated diagnosis of melanoma or skin cancers in the primary care setting met the review inclusion criteria. Kassianos et $a \tilde{l}^{1}$ reviewed 39 smartphone applications and found little evidence of clinical or research-based input into the design or evaluation of these apps. A recent editorial in The Lancet Oncology supported this finding, ${ }^{52}$ and urged caution with early adoption of new technologies that are often poorly designed and untested, stressing the need to ensure that these technologies are appropriate, cost-effective and do not compromise patient safety. Recent studies have tested the application of artificial intelligence, neural networks and machine learning to the diagnosis of skin lesions; however, they have not yet been assessed in primary care settings.

\section{Strengths, limitations and future research}

Our review examines the evidence for dermoscopy use in the primary care setting. It builds on the recent Cochrane review which explicitly reviewed evidence only about diagnostic accuracy of dermoscopy, with and without naked-eye examination, and describes this in specialist and generalist settings. ${ }^{19}$ We therefore included studies with a range of methods, surveys and qualitative studies, as well as RCTs and diagnostic accuracy studies, but still only identified a relatively small number of publications. Unfortunately, we were unable to perform a meta-analysis due to the heterogeneity in study designs, settings, populations and outcomes. All the studies are from high-income countries and therefore may be less generalisable to other countries with different healthcare systems.

\section{CONCLUSIONS}

Despite the limited evidence, this review provides moderate support for the use of dermoscopy in primary 
care, with the weight of the available evidence pointing to a benefit in diagnostic accuracy for managing suspicious skin lesions. Dermoscopy is acceptable to PCPs, so it could help them triage suspicious lesions for urgent referral or reassurance. However, it will be important to establish further evidence on minimum for training to reach competence, as well as the cost-effectiveness and patient acceptability of implementing dermoscopy in primary care.

Acknowledgements The authors thank Isla Kuhn, Reader Services Librarian, University of Cambridge Medical Library, for her help in developing the search strategy, and Margaret Johnson, a patient advocate, who provided regular comments on the study from its conception.

Contributors 0J developed the protocol, completed the search, screened the articles for inclusion, extracted the data, synthesised the findings, interpreted the results and drafted the manuscript. LJ screened the articles for inclusion, extracted the data and critically revised the manuscript. MvM screened the articles for inclusion, extracted the data and critically revised the manuscript. SH screened the articles for inclusion and critically revised the manuscript. NB developed the protocol and critically revised the manuscript. PH developed the protocol and critically revised the manuscript. JE developed the protocol, interpreted the results and critically revised the manuscript. FMW developed the protocol, synthesised the findings, interpreted the results and critically revised the manuscript. All authors approved the final version.

Funding This research arises from the CanTest Collaborative, which is funded by Cancer Research UK (C8640/A23385), of which FW is Director and JE is Associate Director. This work was also supported by FMW's Clinician Scientist Award (RG 68235) from the National Institute for Health Research (NIHR). The views expressed in this publication are those of the authors and not necessarily those of the National Health Service, the NIHR or the Department of Health. The funding sources had no role in the study design, data collection, data analysis, data interpretation, writing of the report or in the decision to submit for publication.

Competing interests $\mathrm{PH}$ is Clinical Advisor for Skin Cancer to Check4Cancer, a company that offers teledermatoscopic analysis of pigmented skin lesions (https:// www.check4cancer.com), and is a medical advisor to MedX, the Canadian company with the IP for the SIAscope (https://medxhealth.com/default.aspx).

Patient consent for publication Not required.

Provenance and peer review Not commissioned; externally peer reviewed.

Data availability statement Data are available upon reasonable request.

Open access This is an open access article distributed in accordance with the Creative Commons Attribution 4.0 Unported (CC BY 4.0) license, which permits others to copy, redistribute, remix, transform and build upon this work for any purpose, provided the original work is properly cited, a link to the licence is given, and indication of whether changes were made. See: https://creativecommons.org/ licenses/by/4.0/.

\section{REFERENCES}

1. WCRF International. Skin cancer statistics: international world cancer research fund. 2018. Available: https://www.wcrf.org/dietandcancer/ cancer-trends/skin-cancer-statistics [Accessed 05 Feb 2019].

2. Mistry M, Parkin DM, Ahmad AS, et al. Cancer incidence in the United Kingdom: projections to the year 2030. Br J Cancer 2011;105:1795-803.

3. Hiom SC. Diagnosing cancer earlier: reviewing the evidence for improving cancer survival. Br J Cancer 2015;112(Suppl 1):S1-5.

4. NICE. NICE NG14 melanoma assessment \& management.pdf. UK: National Institute for Clinical Excellence, 2015.

5. Chuchu N, Dinnes J, Takwoingi Y, et al. Smartphone applications for the diagnosis of cutaneous melanoma in adults. Cochrane Database Syst Rev. In Press 2018.

6. Dinnes J, Deeks JJ, Chuchu N, et al. Reflectance confocal microscopy for the diagnosis of cutaneous melanoma in adults. Cochrane Database Syst Rev. In Press 2018.

7. Ferrante di Ruffano L, Dinnes J, Deeks JJ, et al. Optical coherence tomography for diagnosing skin cancer in adults. Cochrane Database Syst Rev. In Press 2018;29.

8. Ferrante di Ruffano L, Takwoingi Y, Dinnes J, et al. ComputerAssisted diagnosis techniques (dermoscopy and spectroscopy- based) for diagnosing skin cancer in adults. Cochrane Database Syst Rev. In Press;10.

9. Dinnes J, Bamber J, Chuchu N, et al. High-Frequency ultrasound for diagnosing skin cancer in adults. Cochrane Database Syst Rev. In Press 2018;29.

10. Rosendahl C, Cameron A, McColl I, et al. Dermatoscopy in routine practice - 'Chaos and Clues'. Australian Family Physician 2012;41:482-7.

11. Argenziano G, Cerroni L, Zalaudek I, et al. Accuracy in melanoma detection: a 10-year multicenter survey. J Am Acad Dermatol 2012;67:54-9.

12. Menzies SW, Chamberlain A, Soyer HP, et al. What is the role of dermoscopy in melanoma diagnosis? Sydney: cancer Council Australia: cancer Council Australia melanoma guidelines Working Party, 2018. Available: https://wiki.cancer.org.au/australia/Clinical_ question:What_is_the_role_of_dermoscopy_in_melanoma_ diagnosis\%3F [Accessed cited 30th Aug 2018].

13. Vestergaard ME, Macaskill P, Holt PE, et al. Dermoscopy compared with naked eye examination for the diagnosis of primary melanoma: a meta-analysis of studies performed in a clinical setting. $\mathrm{Br} \mathrm{J}$ Dermatol 2008;159:669-76.

14. Menzies SW, Emery J, Staples M, et al. Impact of dermoscopy and short-term sequential digital dermoscopy imaging for the management of pigmented lesions in primary care: a sequential intervention trial. Br J Dermatol 2009;161:1270-7.

15. Noor O, Nanda A, Rao BK. A dermoscopy survey to assess who is using it and why it is or is not being used. Int J Dermatol 2009;48:951-2.

16. Chamberlain AJ, Kelly JW. Use of dermoscopy in Australia. Med J Aust 2007;187:252-3.

17. Vestergaard ME, Menzies SW. Automated diagnostic instruments for cutaneous melanoma. Semin Cutan Med Surg 2008;27:32-6.

18. Haenssle HA, Fink $C$, Schneiderbauer R, et al. Man against machine: diagnostic performance of a deep learning convolutional neural network for dermoscopic melanoma recognition in comparison to 58 dermatologists. Ann Oncol 2018;29:1836-42.

19. Dinnes J, Deeks JJ, Chuchu N, et al. Cochrane skin cancer diagnostic test accuracy group. Dermoscopy, with and without visual inspection, for the diagnosis of melanoma in adults. Cochrane Database Syst Rev 2018;10.

20. Moher D, Shamseer L, Clarke M, et al. Preferred reporting items for systematic review and meta-analysis protocols (PRISMA-P) 2015 statement. Syst Rev 2015;4:1

21. Jones OT, Jurasceck L, Van Melle M, et al. Can dermoscopy be used accurately and effectively in primary care to appropriately triage possible melanoma? Prospero: CRD42018091395, 2018. Available: https://www.crd.york.ac.uk/prospero/display_record.php?RecordID= 91395 [Accessed 15 Aug 2018].

22. Joanna Briggs Institute UoA. Joanna Briggs Institute critical appraisal tools, 2018. Available: http://joannabriggs.org/ [Accessed 30 May 2018].

23. Argenziano G, Puig S, Zalaudek I, et al. Dermoscopy improves accuracy of primary care physicians to triage lesions suggestive of skin cancer. J Clin Oncol 2006:24:1877-82.

24. Koelink CJL, Vermeulen KM, Kollen BJ, et al. Diagnostic accuracy and cost-effectiveness of dermoscopy in primary care: a cluster randomized clinical trial. J Eur Acad Dermatol Venereol 2014;28:1442-9.

25. Rosendahl C, Williams G, Eley D, et al. The impact of subspecialization and dermatoscopy use on accuracy of melanoma diagnosis among primary care doctors in Australia. J Am Acad Dermatol 2012;67:846-52.

26. Ferrándiz L, Ojeda-Vila T, Corrales A, et al. Internet-Based skin cancer screening using clinical images alone or in conjunction with dermoscopic images: a randomized teledermoscopy trial. J Am Acad Dermatol 2017;76:676-82.

27. Ahmadi K, Prickaerts E, Smeets JGE, et al. Current approach of skin lesions suspected of malignancy in general practice in the Netherlands: a quantitative overview. J Eur Acad Dermatol Venereol 2017;27.

28. Bourne P, Rosendahl C, Keir J, et al. BLINCK-A diagnostic algorithm for skin cancer diagnosis combining clinical features with dermatoscopy findings. DPC 2012;2.

29. Menzies SW, Bischof L, Talbot $\mathrm{H}$, et al. The performance of SolarScan: an automated dermoscopy image analysis instrument for the diagnosis of primary melanoma. Arch Dermatol 2005;141:1388-96.

30. Pagnanelli G, Soyer HP, Argenziano G, et al. Diagnosis of pigmented skin lesions by dermoscopy: web-based training improves diagnostic performance of non-experts. Br J Dermatol 2003;148:698-702. 
31. Rogers T, Marino ML, Dusza SW, et al. A clinical aid for detecting skin cancer: the triage amalgamated dermoscopic algorithm (TadA). J Am Board Fam Med 2016;29:694-701.

32. Rogers T, Marino M, Dusza SW, et al. Triage amalgamated dermoscopic algorithm (TadA) for skin cancer screening. Dermatol Pract Concept 2017;7:39-46.

33. Westerhoff K, McCarthy WH, Menzies SW. Increase in the sensitivity for melanoma diagnosis by primary care physicians using skin surface microscopy. Br J Dermatol 2000;143:1016-20.

34. Börve A, Gyllencreutz J, Terstappen K, et al. Smartphone teledermoscopy referrals: a novel process for improved triage of skin cancer patients. Acta Derm Venereol 2015;95:186-90.

35. Grimaldi L, Silvestri A, Brandi C, et al. Digital epiluminescence dermoscopy for pigmented cutaneous lesions, primary care physicians, and telediagnosis: a useful tool? J Plast Reconstr Aesthet Surg 2009;62:1054-8.

36. Moreno-Ramirez D, Ferrandiz L, Galdeano R, et al. Teledermatoscopy as a triage system for pigmented lesions: a pilot study. Clin Exp Dermatol 2006;31:13-18.

37. van der Heijden JP, Thijssing L, Witkamp L, et al. Accuracy and reliability of teledermatoscopy with images taken by general practitioners during everyday practice. $J$ Telemed Telecare 2013;19:320-5

38. Chappuis P, Duru G, Marchal O, et al. Dermoscopy, a useful tool for general practitioners in melanoma screening: a nationwide survey. $\mathrm{Br}$ J Dermatol 2016;175:744-50.

39. Morris J, Alfonso S, Hernandez N, et al. Examining the factors associated with past and present dermoscopy use among family physicians. Dermatol Pract Concept 2017;7:63-70.

40. Morris J, Alfonso S, Hernandez N, et al. Use of and intentions to use dermoscopy among physicians in the United States. Dermatol Pract Concept 2017;7:7-16

41. Livingstone J, Solomon J. An assessment of the cost-effectiveness, safety of referral and patient satisfaction of a general practice teledermatology service. London J Prim Care 2015;7:31-5.
42. Stratton D, Loescher LJ. The acceptance of mobile teledermoscopy by primary care nurse practitioners in the state of Arizona. J Am Assoc Nurse Pract 2016;28:287-93.

43. Rosendahl C, Tschandl P, Cameron A, et al. Diagnostic accuracy of dermatoscopy for melanocytic and nonmelanocytic pigmented lesions. J Am Acad Dermatol 2011;64:1068-73.

44. Secker L, Buis P, Bergman W, et al. Effect of a dermoscopy training course on the accuracy of primary care physicians in diagnosing pigmented lesions. Acta Derm Venereol 2017;97:263-5.

45. Herschorn A. Dermoscopy for melanoma detection in family practice. Can Fam Physician 2012;58:740-5.

46. Troyanova P. A beneficial effect of a short-term formal training course in epiluminescence microscopy on the diagnostic performance of dermatologists about cutaneous malignant melanoma. Skin Res Technol 2003;9:269-73.

47. Stanganelli let al. Diagnosis of pigmented skin lesions by epiluminescence microscopy determinants of accuracy improvement in a nationwide training programme for practical dermatologists. Public Health 1999;113:237-42.

48. Usher-Smith JA, Sharp SJ, Griffin SJ. The spectrum effect in tests for risk prediction, screening, and diagnosis. BMJ 2016;353.

49. Wilson ECF, Emery JD, Louise Kinmonth A, et al. The costeffectiveness of a novel siascopic diagnostic aid for the management of pigmented skin lesions in primary care: a decision-analytic model. Value in Health 2013;16:356-66.

50. Walter FM, Morris HC, Humphrys E, et al. Effect of adding a diagnostic aid to best practice to manage suspicious pigmented lesions in primary care: randomised controlled trial. BMJ 2012;345:e4110.

51. Kassianos AP, Emery JD, Murchie P, et al. Smartphone applications for melanoma detection by community, patient and generalist clinician users: a review. British Journal of Dermatology 2015;172:1507-18

52. The Lancet Oncology. Digital oncology apps: revolution or evolution? Lancet Oncol 2018;19:999. 